\title{
Hypergeometric solutions of some algebraic equations *
}

\author{
A.M. Perelomov ${ }^{\dagger}$ \\ Max-Planck-Institut für Mathematik, \\ Vivatsgasse 7, D-53111 Bonn, Germany
}

To the memory of A.N. Tyurin

\begin{abstract}
We give the hypergeometric solutions of some algebraic equations including the general fifth degree equation.
\end{abstract}

1. It is well known that the general algebraic equation of degree $n \geq 5$ cannot be solved by radicales [Ab 1826]. However, it may be solved if we use wider classes of functions. For example, the fifth degree equation may be solved in modular functions [He 1858], [Kr 1858], [Kl 1884]; the general algebraic equation may be solved in hyperelliptic theta constants [Um 1984].

Another approach consists in the consideration of the algebraic solutions of the differential equations and was used in the classical Schwarz's paper [Sch 1873], where all algebraic solutions of the standard hypergeometric equation were found. It was intensively developed by Poincaré.

The results of Schwarz were completed in the paper [BH 1989] by the classification of the algebraic generalized hypergeometric functions ${ }_{n} F_{n-1}$.

* This paper aroused from the problem of normalization of the ground state wave function of the trigonometric $n$-particle Calogero-Sutherland system (see the review paper [OP 1983]). The relation of this problem with the results of the present note will be considered later.

${ }^{\dagger}$ On leave of absence from Institute for Theoretical and Experimental Physics, 117259 Moscow, Russia. Current e-mail address: perelomo@dftuz.unizar.es 
There is one series corresponding to the case under consideration, and the 76 exceptional cases. Note that for $n \geq 9$ there are no exceptional cases.

In [BH 1989] the monodromy groups for these functions were calculated explicitly. They are the groups generated by the complex reflections in $\mathbb{C}^{n}$. The differential Galois groups of the corresponding hypergeometric differential equations are also explicitly calculated there. However, the corresponding algebraic equations were not calculated.

In this note we give the explicit solution of the equations of type

$$
\begin{aligned}
F_{n}(x, t) & \equiv x^{n}-x+t=0, \quad n>1, \\
G_{n}(y, t) & \equiv \gamma_{n} t^{n-1} y^{n}-(y-1)\left(y+\frac{1}{n-1}\right)^{n-1}=0, \quad \gamma_{n}=\frac{n^{n}}{(n-1)^{n-1}}
\end{aligned}
$$

in the class of hypergeometric functions of type

$$
{ }_{n-1} F_{n-2}\left(\alpha_{1}, \ldots, \alpha_{n-1} ; \beta_{1}, \ldots, \beta_{n-2} ; \gamma t^{n-1}\right) .
$$

Note that the general equations of degrees 2,3,4 and 5 may be reduced to equation (1) by Tschirnhaus transformation, see [Fr 1924] ${ }^{1}$.

2. First, let us remark that the normalization of the ground state wave function of the $n$-particle Calogero-Sutherland problem is equivalent to the calculation of the integral

$$
\int_{0}^{\pi} \cdots \int_{0}^{\pi}\left\{\prod_{1 \leq j<l \leq n} \sin \left(q_{j}-q_{l}\right)\right\}^{k} d q_{1} \cdots d q_{n} .
$$

In the process, there appear binomial coefficients of special type

$$
c_{k}^{(n, j)}=\left(\begin{array}{c}
n k+j \\
k
\end{array}\right)=\frac{(n k+j) !}{((n-1) k+j) ! k !} .
$$

It is natural to introduce for them the generating functions

$$
H_{n, j}(z)=\sum_{k=0}^{\infty} c_{k}^{(n, j)} z^{k}, \quad n \geq 2, \quad j=0,1, \ldots
$$

\footnotetext{
${ }^{1}$ After this work had been completed, I learned from Prof. Zagier that the solution of equation (1) is also known to him, unpublished.
} 
Let us introduce also the functions

$$
\begin{aligned}
& y_{n, j}(t)=t^{j} H_{n, j}(z), \quad \text { where } \quad z=t^{n-1}, \quad j=0,1, \ldots \\
& x_{n, j}(t)=j \int_{0}^{t} y_{n, j-1}(\tau) d \tau, \quad j=1,2, \ldots
\end{aligned}
$$

These functions have a number of nice properties, in particular, $x_{n, 1}(t)$ and $y_{n, 0}(t)$ are the solutions of equations (1) and (2).

Recall the definition of the generalized hypergeometric function:

$$
\begin{aligned}
& { }_{m} F_{n}\left(\alpha_{1}, \alpha_{2}, \ldots, \alpha_{m} ; \beta_{1}, \beta_{2}, \ldots \beta_{n} ; \gamma z\right)=\sum_{k=0}^{\infty} c_{k} z^{k}= \\
& \sum_{k=0}^{\infty} \frac{\left(\alpha_{1}\right)_{k}\left(\alpha_{2}\right)_{k} \cdots\left(\alpha_{m}\right)_{k}}{\left(\beta_{1}\right)_{k}\left(\beta_{2}\right)_{k} \cdots\left(\beta_{n}\right)_{k} k !}(\gamma z)^{k},
\end{aligned}
$$

where

$$
(\alpha)_{k}=\alpha(\alpha+1) \cdots(\alpha+k-1)
$$

is the Pochhammer symbol.

Lemma 1. The functions (6)-(8) are algebraic and of hypergeometric type. Namely,

$$
H_{n, j}(z)={ }_{n-1} F_{n-2}\left(\frac{j+1}{n}, \ldots, \frac{j+n}{n} ; \frac{j+1}{n-1}, \ldots, \frac{j+n-1}{n-1} ; \gamma_{n} z\right),
$$

where

$$
\gamma_{n}=\frac{n^{n}}{(n-1)^{n-1}}
$$

The functions $y_{n, j}(t)$ are given by formula (7). We have also

$$
\begin{aligned}
& x_{n, j}(t)= \\
& t^{j}{ }_{n-1} F_{n-2}\left(\frac{j}{n}, \frac{j+1}{n}, \ldots, \frac{j+n-1}{n} ; \frac{j+1}{n-1}, \frac{j+2}{n-1}, \ldots, \frac{j+n-1}{n-1} ; \gamma_{n} z\right) .
\end{aligned}
$$

Proof. This follows from the fact that for the generalized hypergeometric function (9)

$$
\frac{c_{k+1}}{c_{k}}=\frac{\left(k+\alpha_{1}\right)\left(k+\alpha_{2}\right) \cdots\left(k+\alpha_{m}\right)}{\left(k+\beta_{1}\right)\left(k+\beta_{2}\right) \cdots\left(k+\beta_{n}\right)} \gamma
$$


and we have the identities

$$
\begin{aligned}
& { }_{n} F_{n-1}\left(\alpha_{1}, \ldots, \alpha_{n-1}, \alpha ; \beta_{1}, \ldots, \beta_{n-2}, \alpha ; z\right)= \\
& { }_{n-1} F_{n-2}\left(\alpha_{1}, \ldots, \alpha_{n-1} ; \beta_{1}, \ldots, \beta_{n-2} ; z\right) .
\end{aligned}
$$

The algebraic character of the functions $H_{n, j}(z)$ becomes evident after the comparison of (10) with the list of functions from [BH 1989].

The hypergeometric character of these functions gives the possibility to easily differentiate and integrate them. For this we may use the well-known formulae:

$$
\begin{aligned}
& \frac{d}{d z}{ }_{m} F_{n}\left(\alpha_{1}, \ldots, \alpha_{m} ; \beta_{1}, \ldots, \beta_{n} ; z\right)= \\
& \frac{\alpha_{1} \cdots \alpha_{m}}{\beta_{1} \cdots \beta_{n}}{ }_{m} F_{n}\left(\alpha_{1}+1, \ldots, \alpha_{m}+1 ; \beta_{1}+1, \ldots, \beta_{n}+1 ; z\right), \quad \text { (15) } \\
& \int{ }_{m} F_{n}\left(\alpha_{1} \cdots \alpha_{m} ; \beta_{1} \cdots \beta_{n} ; z\right) d z= \\
& \frac{\left(\beta_{1}-1\right) \cdots\left(\beta_{n}-1\right)}{\left(\alpha_{1}-1\right) \cdots\left(\alpha_{m}-1\right)}{ }_{m} F_{n}\left(\alpha_{1}-1, \cdots, \alpha_{m}-1 ; \beta_{1}-1, \cdots, \beta_{n}-1 ; z\right)+\text { const. }
\end{aligned}
$$

From these formulae we obtain

$$
\begin{aligned}
& \frac{d}{d z} H_{n, j}(z)= \\
& (j+n)_{n-1} F_{n-2}\left(\frac{j+n+1}{n}, \ldots, \frac{j+2 n}{n} ; \frac{j+n}{n-1}, \ldots, \frac{j+2 n-2}{n-1} ; \gamma_{n} z\right)
\end{aligned}
$$

and formula (12).

3. Note that equation (1) has the solution $x(t), x(0)=0$, analytic in the disc $D_{r_{n}}$ of some radius $r_{n}>0$. Consider also the function $y(t)=x^{\prime}(t)$. Differentiating (1) with respect to $t$, we obtain

$$
\left(1-n x^{n-1}\right) y=1
$$


Equation (1) yields also

$$
x^{n-1}=\frac{1}{n}\left(1-\frac{1}{y}\right)
$$

From this it follows equation (2).

The main result of this paper is

Theorem 1. The solutions of equations (1) and (2) are given by the formulae

$$
x(t)=x_{n, 1}(t), \quad y(t)=y_{n, 0}(t) .
$$

We also have

$$
(x(t))^{j}=x_{n, j}(t), \quad(x(t))^{j} y(t)=y_{n, j}(t) .
$$

4. Before to prove this theorem, we give some properties of generalized hypergeometric functions (for more details see [Po 1888] and [BE 1953]).

The generalized hypergeometric function ${ }_{n} F_{n-1}(z)$ is defined by the series

$$
{ }_{n} F_{n-1}\left(\alpha_{1}, \ldots, \alpha_{n} ; \beta_{1}, \ldots, \beta_{n-1} ; z\right)=\sum_{k=0}^{\infty} \frac{\left(\alpha_{1}\right)_{k} \cdots\left(\alpha_{n}\right)_{k}}{\left(\beta_{1}\right)_{k} \cdots\left(\beta_{n-1}\right)_{k} k !} z^{k}
$$

which converges in the unit disc.

The function $u(z)={ }_{n} F_{n-1}(z)$ satisfies the hypergeometric equation

$$
D \cdot\left(D+\beta_{1}-1\right) \cdots\left(D+\beta_{n-1}-1\right) u-z\left(D+\alpha_{1}\right) \cdots\left(D+\alpha_{n}\right) u=0
$$

with $D=z d / d z$. This function is analytic on the Riemann sphere $\mathbb{C} P^{1}=$ $\mathbb{C}^{1} \cup\{\infty\}$ except the points $z=0, z=\infty$ and $z=1$. The local behaviour of this function at these points is defined by the exponents

$$
\begin{array}{ll}
1-\beta_{1}, \ldots, 1-\beta_{n-1}, 0 & \text { at } z=0, \\
\alpha_{1}, \ldots, \alpha_{n} & \text { at } z=\infty, \\
0,1, \ldots, n-2, \quad \gamma=\sum_{j=1}^{n-1} \beta_{j}-\sum_{l=1}^{n} \alpha_{l} & \text { at } \quad z=1 .
\end{array}
$$

If the numbers $\beta_{1}, \ldots, \beta_{n-1}$ are distinct $\bmod \mathbb{Z}$, then $n$ independent solutions of equation (21) are given by the formula

$$
z^{1-\beta_{i}}{ }_{n} F_{n-1}\left(1+\alpha_{1}-\beta_{i}, \ldots, 1+\alpha_{n}-\beta_{i} ; 1+\beta_{1}-\beta_{i}, \ldots, 1+\beta_{n}-\beta_{i} ; z\right),
$$


where $i=1, \ldots, n, \beta_{n}=1$, and the $\operatorname{sign} . \hat{\text {. denotes that the argument }}$ $1+\beta_{i}-\beta_{i}$ is omitted.

In [Po 1888] the following proposition was proved.

Lemma 2. [Po 1888]. If in (24) $\gamma \notin \mathbb{N}$ then near the point $z=1$ equation (23) has $n-1$ analytic solutions of the form

$$
u_{j}(z)=(z-1)^{j-1}+O\left((z-1)^{n-1}\right)
$$

for $j=1, \ldots, n-1$ corresponding to the exponents $0,1, \ldots, n-2$.

Now we are ready to prove

Theorem 2. The function $y(t)=x^{\prime}(t)$ has the following properties:

1. $y(t)$ is analytic in the disc

$$
D_{r_{n}}=\left\{t:|t|<r_{n}\right\}, \quad \text { where } \quad r_{n}^{n-1}=\frac{(n-1)^{n-1}}{n^{n}},
$$

and the expansion of $y(t)$ as $t \rightarrow 0$ is of the form

$$
y(t)=\tilde{y}(z)=1+c_{1} z+c_{2} z^{2}+\cdots, \quad \text { where } \quad z=t^{n-1} .
$$

2. The function $\tilde{y}(z)$ is analytic on $\mathbb{C} P^{1}$ and its values are distinct from zero and infinity except at points $z=z_{0}=r_{n}^{n-1}=\gamma_{n}^{-1}$ and $z=\infty$. At $z=z_{0}$ it is equal to infinity, and at $z=\infty$ it vanishes.

As $|t| \rightarrow \infty$, we have

$$
x(t)=t \sum_{k=1}^{n-1} z^{-\alpha_{k}} g_{k}(z), \quad \tilde{y}(z)=\sum_{k=1}^{n-1} z^{-\alpha_{k}} \tilde{g}_{k}(z), \quad \alpha_{k}=\frac{k}{n}, \quad z=t^{n-1} .
$$

At $z=\infty$, the functions $g_{k}(z)$ and $\tilde{g}_{k}(z)$ are analytic and their values are distinct from zero and infinity.

3. Near the point $z=z_{0}$, the function $\tilde{y}(z)$ has the form

$$
\tilde{y}(z)=\left(z-z_{0}\right)^{-1 / 2} f_{1}(z)+f_{2}(z),
$$

where the functions $f_{1}(z)$ and $f_{2}(z)$ are analytic at $z=z_{0}$ with $f_{1}\left(z_{0}\right) \neq$ 0 and $f_{2}\left(z_{0}\right) \neq \infty$. 
4. The dimension of the linear space of all branches of the function $\tilde{y}(z)$ obtained by going around the branch points $z=z_{0}$ and $z=\infty$ is equal to $n-1$.

Proof.

1. The first statement of theorem follows from formulae (18), (19) and from the solution of equation (1) by iterations

$$
\begin{aligned}
x & =t+x^{n}, \\
x & =t+\left(t+x^{n}\right)^{n}, \quad x=t+\left(t+\left(t+x^{n}\right)^{n}\right)^{n}, \ldots, \\
x & =t+\frac{c_{1}}{n} t^{n}+\frac{c_{2}}{2 n-1} t^{2 n-1}+\frac{c_{3}}{3 n-2} t^{3 n-2}+\cdots, \\
c_{1}=n, \quad & c_{2}=\frac{(2 n)(2 n-1)}{1 \cdot 2}, \quad c_{3}=\frac{3 n(3 n-1)(3 n-2)}{1 \cdot 2 \cdot 3}, \cdots .
\end{aligned}
$$

The radius of convergence of this series is determined by the condition that the discriminant of equation (1) vanishes. From this we derive the formula

$$
z_{0}=r_{n}^{n-1}=\gamma_{n}^{-1}=\frac{(n-1)^{n-1}}{n^{n}} .
$$

2. This follows from the iterations of formula

$$
x(t)=\varepsilon_{n} t^{1 / n}\left(1-\frac{x}{t}\right)^{1 / n}, \quad \text { where } \quad \varepsilon_{n}=\exp \left(i \frac{\pi}{n}\right), \quad \text { as } \quad|t| \rightarrow \infty .
$$

3. In the same way, we obtain the expansion of $\tilde{y}(z)$ at the point $z=z_{0}$.

4. Equation (1) has $n$ solutions $x^{(j)}(t), j=1, \ldots, n, x^{(1)}(t)=x(t)$, and we have $\sum x^{(j)}(t)=0$.

Therefore, the $n$ branches $\tilde{y}^{(1)}(z), \ldots, \tilde{y}^{(n)}(z)$ of the algebraic function $\tilde{y}(z)=x^{\prime}(t)$ are linearly dependent

$$
\tilde{y}^{(1)}(z)+\tilde{y}^{(2)}(z)+\cdots+\tilde{y}^{(n)}(z)=0,
$$

and the dimension of the linear space of all branches of the function $\tilde{y}(z)$ is equal to $n-1$.

Now we are ready to prove Theorem 1.

We give two different proofs of this theorem. The first proof is based on the general idea of a beautiful Riemann's paper [Rie 1857] adapted to the problem under consideration. The second one uses the standard calculation of the residue. 
Proof 1. From equations (1) and (2) it follows that the function $\tilde{y}(z)=$ $\left(1-n x^{n-1}\right)^{-1}, z=t^{n-1}$, vanishes only at $z=\infty$, and it is equal to infinity only at $z=z_{0}$. Let $\tilde{y}^{(1)}(z), \ldots, \tilde{y}^{(n)}(z)$ be all the $n$ branches of this function. Then

$$
\tilde{y}^{(1)}(z)+\tilde{y}^{(2)}(z)+\cdots+\tilde{y}^{(n)}(z)=0,
$$

and the space $L_{n}$ of linear combinations of such functions is $(n-1)$-dimensional. At the points $z=0, z=z_{0}$ and $z=\infty$, the expansion of the function $f(z) \in L_{n}$ is defined by the exponents:

$$
\begin{aligned}
\alpha_{1}=\frac{1}{n}, \ldots, \alpha_{n-1}=\frac{n-1}{n}, & \text { at } \quad z=\infty, \\
\beta_{1}=\frac{1}{n-1}, \ldots, \beta_{n-2}=\frac{n-2}{n-1}, & \text { at } \quad z=0, \\
\gamma_{1}=0, \gamma_{2}=1, \ldots, \gamma_{n-2}=n-3, \gamma_{n-1}=-\frac{1}{2} & \text { at } \quad z=z_{0} .
\end{aligned}
$$

On the other hand, $n-1$ linearly independent solutions, $u_{1}(z), \ldots u_{n-1}(z)$, of the hypergeometric equation

$$
D \cdot\left(D+\beta_{1}-1\right) \cdots\left(D+\beta_{n-2}-1\right) u(z)-z\left(D+\alpha_{1}\right) \cdots\left(D+\alpha_{n-1}\right) u(z)=0
$$

with $D=z d / d z$ have the same exponents and satisfy the conditions of the Riemann theorem. Therefore, according to this theorem

$$
\tilde{y}(z)=\tilde{y}^{(1)}(z) \in L_{n}, \quad \tilde{y}(z)=c_{1} u_{1}(z)+\cdots+c_{n-1} u_{n-1}(z) .
$$

The constants $c_{k}$ may be found from the consideration of the limit as $z \rightarrow 0$. As a result, we obtain formulae (20) and $(21)^{2}$.

Proof 2. Here we use the calculation of the residue. We have

$$
\tilde{y}(z)=y_{n, 0}(z)=1+c_{1}^{(0)} z+\cdots+c_{k}^{(0)} z^{k}+\cdots, \quad z=t^{n-1} .
$$

Therefore,

$$
\frac{c_{k}^{(0)}}{t}+\frac{d f_{k}}{d t}=\frac{\tilde{y}(z)}{x^{1+k(n-1)}\left(1-x^{(n-1)}\right)^{1+k(n-1)}},
$$

\footnotetext{
${ }^{2}$ Note that other branches of functions $y^{(j)}(t)$ and $x^{(j)}(t) \in L_{n}$, i.e., they are also the linear combinations of the functions $u_{1}(z), \ldots, u_{n-1}(z)$.
} 
or

$$
\frac{c_{k}^{(0)}}{t}+\frac{d f_{k}}{d t}=\frac{C_{k}^{(0)}}{x}+\frac{d F_{k}}{d x} .
$$

Here $f_{k}(t)$ and $F_{k}(x)$ are some rational functions.

The quantity $C_{j}^{(0)}$ is given by the expansion of the function

$$
(1-z)^{-(1+k(n-1))}=\sum_{j=0}^{\infty} C_{j}^{(0)} z^{j}
$$

Hence,

$$
c_{k}^{(0)}=C_{k}^{(0)}=\left(\begin{array}{c}
k n \\
k
\end{array}\right) .
$$

The analogous calculation for the function

$$
y_{n, j}(z)=t^{j} \sum_{k=0}^{\infty} c_{k}^{(j)} z^{k}
$$

gives

$$
c_{k}^{(j)}=\left(\begin{array}{c}
k n+j \\
k
\end{array}\right) .
$$

This completes the proof.

From this it follows formula $(7)$ for $y_{n, j}(t)$. Integrating $y_{n, j-1}(t)$ we obtain (21).

So, Theorem 1 is proved.

5. We give here the additional properties of the functions $x_{n, j}$ and $y_{n, j}$.

Lemma 3. The function $y(t)=y_{n, 0}(t)$ satisfies the following nonlinear differential equation

$$
\frac{1}{y} \frac{d}{d t} \frac{1}{y} \frac{d}{d t} \cdots \frac{1}{y}=-n ! .
$$

Proof. We have

$$
\frac{1}{y}=1-n x^{n-1}
$$

Differentiating this $n-1$ times with respect to $t$, we obtain equation (46). 
Lemma 4. For the function $y(t)$ there is the following formal integral representation ${ }^{3}$

$$
y(t)=\int_{\mathbb{C}} d \mu(z, \bar{z}) \exp \left(\bar{z}(z+t)^{n}\right),
$$

where

$$
d \mu(z, \bar{z})=\frac{1}{\pi} \exp \left(-|z|^{2}\right) d z d \bar{z} .
$$

Proof. We can check this by expanding of the integrand in the series and using the formula

$$
\int d \mu(z, \bar{z}) z^{j} \bar{z}^{k}=\delta_{j k} k !
$$

In conclusion, observe that from the evident formulae such as

$$
(x(t))^{j}(x(t))^{l}=(x(t))^{j+l}, \quad(x(t))^{j}\left(x(t)^{l} y(t)\right)=(x(t))^{j+l} y(t)
$$

there follows nontrivial (and probably new) identities for the generalized hypergeometric functions. We give here a couple of them.

Lemma 5. The following identities are valid:

$$
\begin{aligned}
& { }_{n} F_{n-1}\left(\frac{j}{n}, \ldots, \frac{j+n-1}{n} ; \frac{j+1}{n-1}, \ldots, \frac{j+n-1}{n-1} ; z\right) \\
& \times \quad{ }_{n} F_{n-1}\left(\frac{l}{n}, \ldots, \frac{l+n-1}{n} ; \frac{l+1}{n-1}, \ldots, \frac{l+n-1}{n-1} ; z\right)= \\
& { }_{n} F_{n-1}\left(\frac{j+l}{n}, \ldots, \frac{j+l+n-1}{n} ; \frac{j+l+1}{n-1}, \ldots, \frac{j+l+n-1}{n-1} ; z\right), \\
& { }_{n} F_{n-1}\left(\frac{j}{n}, \ldots, \frac{j+n-1}{n} ; \frac{j+1}{n-1}, \ldots, \frac{j+n-1}{n-1} ; z\right) \\
& \times \quad{ }_{n-1} F_{n-2}\left(\frac{l+1}{n}, \ldots, \frac{l+n}{n} ; \frac{l+1}{n-1}, \ldots, \frac{l+n-1}{n-1} ; z\right)= \\
& { }_{n-1} F_{n-2}\left(\frac{j+l}{n}, \ldots, \frac{j+l+n}{n} ; \frac{l+l+1}{n-1}, \ldots, \frac{j+l+n-1}{n-1} ; z\right) .
\end{aligned}
$$

${ }^{3}$ The term formal means that we should first expand the function $\exp \left(\bar{z}(z+t)^{n}\right)$ in the power series and only then integrate it. Note that for the functions $x_{n, j}(t)$ and $y_{n, j}(t)$ we also have the standard integral representations (see [Po 1888], [BE 1953]). 


\section{Appendix. Explicit expressions for functions $x(t)=x_{n, 1}(t)$ and $y_{j}(t)=y_{n, j}(t)$ for $\mathbf{n}=\mathbf{2}, \mathbf{3}, \mathbf{4}, \mathbf{5}$ and 6.}

$n=2$

$$
\begin{aligned}
x(t)= & t_{2} F_{1}\left(\frac{1}{2}, 1 ; 2 ; 4 t\right), \\
x(t)= & t+t^{2}+2 t^{3}+5 t^{4}+2 \cdot 7 t^{5}+2 \cdot 3 \cdot 7 t^{6}+2^{2} \cdot 3 \cdot 11 t^{7} \\
+ & 3 \cdot 11 \cdot 13 t^{8}+2 \cdot 5 \cdot 11 \cdot 13 t^{9}+\cdots \\
y_{0} & ={ }_{1} F_{0}\left(\frac{1}{2} ; 4 t\right), \\
y_{0} & =1+2 t+2 \cdot 3 t^{2}+2^{2} \cdot 5 t^{3} \\
& +2 \cdot 5 \cdot 7 t^{4}+2^{2} \cdot 3^{2} \cdot 7 t^{5}+2^{2} \cdot 3 \cdot 7 \cdot 11 t^{6} \\
& +2^{3} \cdot 3 \cdot 11 \cdot 13 t^{7}+2 \cdot 3^{2} \cdot 5 \cdot 11 \cdot 13 t^{8}+\cdots \\
y_{1} & =t+3 t^{2}+2 \cdot 5 t^{3}+5 \cdot 7 t^{4}+2 \cdot 3^{2} \cdot 7 t^{5}+\cdots
\end{aligned}
$$

$$
\begin{aligned}
& n=3 \\
& \qquad \begin{aligned}
x(t)= & t_{2} F_{1}\left(\frac{1}{3}, \frac{2}{3} ; \frac{3}{2} ; \frac{27}{4} t^{2}\right) \\
x(t)= & t+t^{3}+3 t^{5}+2^{2} \cdot 3 t^{7}+5 \cdot 11 t^{9}+3 \cdot 7 \cdot 13 t^{11} \\
& +2^{2} \cdot 3 \cdot 7 \cdot 17 t^{13}+2^{3} \cdot 3 \cdot 17 \cdot 19 t^{15}+3^{2} \cdot 11 \cdot 19 \cdot 23 t^{17}+\cdots \\
y_{0}(t) & ={ }_{2} F_{1}\left(\frac{1}{3}, \frac{2}{3} ; \frac{1}{2} ; \frac{27}{4} t^{2}\right), \\
y_{0}(t) & =y(t \mid 3,0)=1+3 t^{2}+3 \cdot 5 t^{4}+2^{2} \cdot 3 \cdot 7 t^{6}+3^{2} \cdot 5 \cdot 11 t^{8} \\
& +3 \cdot 7 \cdot 11 \cdot 13 t^{10}+2^{2} \cdot 3 \cdot 7 \cdot 13 \cdot 17 t^{12} \\
& +2^{3} \cdot 3^{2} \cdot 5 \cdot 17 \cdot 19 t^{14}+3^{2} \cdot 11 \cdot 17 \cdot 19 \cdot 23 t^{16}+\cdots \\
y_{0}^{-1}(t) & =1-3 t^{2}-2 \cdot 3 t^{4}-3 \cdot 7 t^{6}-2 \cdot 3^{2} \cdot 5 t^{8}-3 \cdot 11 \cdot 13 t^{10} \\
& -2^{3} \cdot 3 \cdot 7 \cdot 13 t^{12}-\cdots
\end{aligned}
\end{aligned}
$$




$$
\begin{aligned}
y_{1}(t) & =t_{2} F_{1}\left(\frac{2}{3}, \frac{4}{3} ; \frac{3}{2} ; \frac{27}{4} t^{2}\right) \\
y_{1}(t) & =t+2^{2} t^{3}+3 \cdot 7 t^{5}+2^{3} \cdot 3 \cdot 5 t^{7}+5 \cdot 11 \cdot 13 t^{9} \\
& +2^{4} \cdot 3 \cdot 7 \cdot 13 t^{11}+2^{2} \cdot 3 \cdot 7 \cdot 17 \cdot 19 t^{13}+\cdots \\
y_{2}(t) & =t^{2}{ }_{3} F_{2}\left(1, \frac{4}{3}, \frac{5}{3} ; \frac{3}{2}, 2 ; \frac{3^{3}}{2^{2}} t^{2}\right) \\
y_{2}(t) & =t^{2}+5 t^{4}+2^{2} \cdot 7 t^{6}+3 \cdot 5 \cdot 11 t^{8} \\
& +7 \cdot 11 \cdot 13 t^{10}+\cdots
\end{aligned}
$$

$n=4$

$$
\begin{aligned}
x(t)= & t_{3} F_{2}\left(\frac{1}{4}, \frac{1}{2}, \frac{3}{4} ; \frac{2}{3}, \frac{4}{3} ; \frac{4^{4}}{3^{3}} t^{3}\right) \\
x(t)= & t+t^{4}+2^{2} t^{7}+2 \cdot 11 t^{10}+2^{2} \cdot 5 \cdot 7 t^{13} \\
+ & 3 \cdot 17 \cdot 19 t^{16}+2^{2} \cdot 7 \cdot 11 \cdot 23 t^{19}+2^{2} \cdot 3^{2} \cdot 5 \cdot 13 \cdot 23 t^{22} \\
+ & 2^{2} \cdot 3^{2} \cdot 13 \cdot 29 \cdot 31 t^{25}+\cdots \\
y_{0}(t)= & { }_{3} F_{2}\left(\frac{1}{4}, \frac{1}{2}, \frac{3}{4} ; \frac{1}{3}, \frac{2}{3} ; \frac{4^{4}}{3^{3}} t^{3}\right) \\
y_{0}(t)= & 1+2^{2} t^{3}+2^{2} \cdot 7 t^{6}+2^{2} \cdot 5 \cdot 11 t^{9}+2^{2} \cdot 5 \cdot 7 \cdot 13 t^{12} \\
+ & 2^{4} \cdot 3 \cdot 17 \cdot 19 t^{15}+2^{2} \cdot 7 \cdot 11 \cdot 19 \cdot 23 t^{18} \\
+ & 2^{3} \cdot 3^{2} \cdot 5 \cdot 11 \cdot 13 \cdot 23 t^{21}+2^{2} \cdot 3^{2} \cdot 5^{2} \cdot 13 \cdot 29 \cdot 31 t^{24}+\cdots \\
& y_{1}(t)=t_{3} F_{2}\left(\frac{1}{2}, \frac{3}{4}, \frac{5}{4} ; \frac{2}{3}, \frac{4}{3} ; \frac{4^{4}}{3^{3}} t^{3}\right) \\
& y_{1}(t)=t+5 t^{4}+2^{2} \cdot 3^{2} t^{7}+2 \cdot 11 \cdot 13 t^{10} \\
& +2^{2} \cdot 5 \cdot 7 \cdot 17 t^{13}+\cdots \\
& +2_{2}(t)=t^{2}{ }_{3} F_{2}\left(\frac{3}{4}, \frac{5}{4}, \frac{3}{2} ; \frac{4}{3}, \frac{5}{3} ; \frac{4^{4}}{3^{3}} t^{3}\right) \\
& y_{2}(t)=t^{2}+2 \cdot 3 t^{5}+3^{2} \cdot 5 t^{8}+2^{2} \cdot 7 \cdot 13 t^{11} \\
&
\end{aligned}
$$




$$
\begin{aligned}
y_{3}(t) & =t^{3}{ }_{4} F_{3}\left(1, \frac{5}{4}, \frac{3}{2}, \frac{7}{4} ; \frac{4}{3}, \frac{5}{3}, 2 ; \frac{4^{4}}{3^{3}} t^{3}\right) \\
y_{3}(t) & =t^{3}+7 t^{6}+5 \cdot 11 t^{9}+5 \cdot 7 \cdot 13 t^{12} \\
& +2^{2} \cdot 3 \cdot 17 \cdot 19 t^{15}+\cdots
\end{aligned}
$$

$n=5$

$$
\begin{aligned}
& x(t)=t_{4} F_{3}\left(\frac{1}{5}, \frac{2}{5}, \frac{3}{5}, \frac{4}{5} ; \frac{1}{2}, \frac{3}{4}, \frac{5}{4} ; \frac{5^{5}}{4^{4}} t^{4}\right) \\
& x(t)=t+t^{5}+5 t^{9}+5 \cdot 7 t^{13}+3 \cdot 5 \cdot 19 t^{17}+2 \cdot 5 \cdot 11 \cdot 23 t^{21} \\
& +3^{2} \cdot 7 \cdot 13 \cdot 29 t^{25}+2^{3} \cdot 5 \cdot 11 \cdot 17 \cdot 31 t^{29} \\
& +3 \cdot 5 \cdot 13 \cdot 17 \cdot 19 \cdot 37 t^{33}+\cdots \\
& y_{0}(t)={ }_{4} F_{3}\left(\frac{1}{5}, \frac{2}{5}, \frac{3}{5}, \frac{4}{5} ; \frac{1}{4}, \frac{1}{2}, \frac{3}{4} ; \frac{5^{5}}{4^{4}} t^{4}\right) \\
& y_{0}(t)=1+5 t^{4}+3^{2} \cdot 5 t^{8}+5 \cdot 7 \cdot 13 t^{12}+3 \cdot 5 \cdot 17 \cdot 19 t^{16} \\
& +2 \cdot 3 \cdot 5 \cdot 7 \cdot 11 \cdot 23 t^{20}+3^{2} \cdot 5^{2} \cdot 7 \cdot 13 \cdot 29 t^{24} \\
& +2^{3} \cdot 5 \cdot 11 \cdot 17 \cdot 29 \cdot 31 t^{28} \\
& +3^{2} \cdot 5 \cdot 11 \cdot 13 \cdot 17 \cdot 19 \cdot 37 t^{32}+\cdots \\
& y_{1}(t)=t_{4} F_{3}\left(\frac{2}{5}, \frac{3}{5}, \frac{4}{5}, \frac{6}{5} ; \frac{1}{2}, \frac{3}{4}, \frac{5}{4} ; \frac{5^{5}}{4^{4}} t^{4}\right) \\
& y_{1}(t)=t+2 \cdot 3 t^{5}+5 \cdot 11 t^{9}+2^{4} \cdot 5 \cdot 7 t^{13} \\
& +3^{2} \cdot 5 \cdot 7 \cdot 19 t^{17}+\cdots \\
& y_{2}(t)=t^{2}{ }_{4} F_{3}\left(\frac{3}{5}, \frac{4}{5}, \frac{6}{5}, \frac{7}{5} ; \frac{3}{4}, \frac{5}{4}, \frac{3}{2} ; \frac{5^{5}}{4^{4}} t^{4}\right) \\
& y_{2}(t)=t^{2}+7 t^{6}+2 \cdot 3 \cdot 11 t^{10}+2^{3} \cdot 5 \cdot 17 t^{14} \\
& +5 \cdot 7 \cdot 11 \cdot 19 t^{18}+2 \cdot 3^{3} \cdot 5 \cdot 13 \cdot 23 t^{22}+\cdots \\
& y_{3}(t)=t^{3}{ }_{4} F_{3}\left(\frac{4}{5}, \frac{6}{5}, \frac{7}{5}, \frac{8}{5} ; \frac{5}{4}, \frac{3}{2}, \frac{7}{4} ; \frac{5^{5}}{4^{4}} t^{4}\right) \\
& y_{3}(t)=t^{3}+2^{3} t^{7}+2 \cdot 3 \cdot 13 t^{11}+2^{4} \cdot 3 \cdot 17 t^{15} \\
& +5 \cdot 7 \cdot 11 \cdot 23 t^{19}+2^{3} \cdot 3^{3} \cdot 5 \cdot 7 \cdot 13 t^{23}+\cdots
\end{aligned}
$$




$$
\begin{aligned}
y_{4}(t) & =t^{4}{ }_{5} F_{4}\left(1, \frac{6}{5}, \frac{7}{5}, \frac{8}{5}, \frac{9}{5} ; \frac{5}{4}, \frac{3}{2}, \frac{7}{4} ; 2 ; \frac{5^{5}}{4^{4}} t^{4}\right) \\
y_{4}(t) & =t^{4}+3^{2} t^{8}+7 \cdot 13 t^{12}+3 \cdot 17 \cdot 19 t^{16} \\
& +2 \cdot 3 \cdot 7 \cdot 11 \cdot 23 t^{20}+3^{2} \cdot 5 \cdot 7 \cdot 13 \cdot 29 t^{24}+\cdots
\end{aligned}
$$

$n=6$

$$
\begin{aligned}
x(t) & =t_{5} F_{4}\left(\frac{1}{6}, \frac{1}{3}, \frac{1}{2}, \frac{2}{3}, \frac{5}{6} ; \frac{2}{5}, \frac{3}{5}, \frac{4}{5}, \frac{6}{5} ; \frac{6^{6}}{5^{5}} t^{5}\right) \\
x(t) & =t+t^{6}+2 \cdot 3 t^{11}+3 \cdot 17 t^{16}+2 \cdot 11 \cdot 23 t^{21} \\
& +3^{3} \cdot 7 \cdot 29 t^{26}+2^{4} \cdot 3 \cdot 7 \cdot 11 \cdot 17 t^{31} \\
& +2 \cdot 13 \cdot 19 \cdot 37 \cdot 41 t^{36}+2 \cdot 3^{2} \cdot 11 \cdot 23 \cdot 43 \cdot 47 t^{41}+\cdots \\
y_{0}(t) & ={ }_{5} F_{4}\left(\frac{1}{6}, \frac{1}{3}, \frac{1}{2}, \frac{2}{3}, \frac{5}{6} ; \frac{1}{5}, \frac{2}{5}, \frac{3}{5}, \frac{4}{5} ; \frac{6^{6}}{5^{5}} t^{6}\right) \\
y_{0}(t) & =1+2 \cdot 3 t^{5}+2 \cdot 3 \cdot 11 t^{10}+2^{4} \cdot 3 \cdot 17 t^{15} \\
& +2 \cdot 3 \cdot 7 \cdot 11 \cdot 23 t^{20}+2 \cdot 3^{3} \cdot 7 \cdot 13 \cdot 29 t^{25} \\
& +2^{4} \cdot 3 \cdot 7 \cdot 11 \cdot 17 \cdot 31 t^{30}+2^{3} \cdot 3^{2} \cdot 13 \cdot 19 \cdot 37 \cdot 41 t^{35} \\
& +2 \cdot 3^{2} \cdot 11 \cdot 23 \cdot 41 \cdot 43 \cdot 47 t^{40}+\cdots
\end{aligned}
$$


Acknowledgments. I am grateful to Professors F. Hirzebruch, D. Leites, A. Levine and Yu.I. Manin for useful remarks and the Max-Planck-Institute für Mathematik, Bonn for hospitality.

\section{References}

[Ab 1826] Abel N.H., Beweis der Unmöglichkeit algebraische Gleichungen von höheren Graden als dem vierten algemein aufzulösen, J. Reine Angew. Math. 1, 65-84

[BE 1953] Bateman H. and Erdélyi A., Higher transcendental functions, Vol.I, New York, McGraw-Hill

[BH 1989] Beukers F. and Heckman G., Monodromy for the hypergeometric function $_{n} F_{n-1}$, Inv. Math. 95, 325-354

[Fr 1924] Fricke R., Lehrbuch der Algebra, Vieweg und Sohn: Braunschweig

[He 1858] Hermite Ch., Sur la résolution de l'équation du cinquième degré, Compt. Rend. 46, 508-515

[Kl 1884] Klein F., Vorlesungen über das Ikosaeder und die Auflösung der Gleichung $5^{\text {ten }}$ Grades, Teubner: Leipzig

[Kr 1858] Kronecker L., Sur la résolution de l'équation du cinquième degré, Compt. Rend. 46, 1150-1152

[OP 1983] Olshanetsky M.A. and Perelomov A.M., Quantum integrable systems related to Lie algberas, Phys. Reps. 94, No.6, 313-404

[Po 1888] Pochhammer L., Zur Theorie der allgemeineren hypergeometrische Reihe, J. Reine Angew. Math. 102, 76-159

[Rie 1857] Riemann B., Beiträge zur Theorie der durch die Gauss'sche Reihe $F(\alpha, \beta, \gamma, x)$ darstellbaren Functionen, Abh. König. Gesell. Wiss. zu Göttingen 7

[Sch 1873] Schwarz H., Über diejenigen Fälle in welchen die Gaussische hypergeometrische Reihe einer algebraische Funktion ihres vierten Elements darstellt. J. Reine Angew. Math. 75, 292-335 
[Um 1984] Umemura H., Resolution of a algebraic equations by theta constants, in: Mumford D., Tata lectures on theta. II., Birkhauser, 3.261 - 3.272 